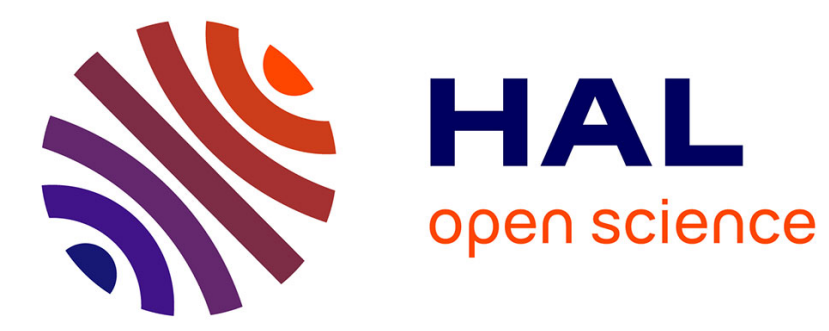

\title{
Proactive Longitudinal Velocity Control In Pedestrians-Vehicle Interaction Scenarios
}

Maria Kabtoul, Philippe Martinet, Anne Spalanzani

\section{To cite this version:}

Maria Kabtoul, Philippe Martinet, Anne Spalanzani. Proactive Longitudinal Velocity Control In Pedestrians-Vehicle Interaction Scenarios. ITSC 2020 - 23rd IEEE International Conference on Intelligent Transportation Systems, Sep 2020, Rhodes, Greece. pp.1-6. hal-02944369

\section{HAL Id: hal-02944369 \\ https://hal.inria.fr/hal-02944369}

Submitted on 21 Sep 2020

HAL is a multi-disciplinary open access archive for the deposit and dissemination of scientific research documents, whether they are published or not. The documents may come from teaching and research institutions in France or abroad, or from public or private research centers.
L'archive ouverte pluridisciplinaire HAL, est destinée au dépôt et à la diffusion de documents scientifiques de niveau recherche, publiés ou non, émanant des établissements d'enseignement et de recherche français ou étrangers, des laboratoires publics ou privés. 


\title{
Proactive Longitudinal Velocity Control In Pedestrians-Vehicle Interaction Scenarios
}

\author{
Maria Kabtoul ${ }^{1,2}$, Philippe Martinet ${ }^{2}$ and Anne Spalanzani ${ }^{1}$
}

\begin{abstract}
Navigation in pedestrian populated environments is a highly challenging task, and a milestone on the way to fully autonomous urban driving systems. Pedestrian populated environments are highly dynamic, uncertain and difficult to predict. The strict safety measures in such environments result in overly reactive navigation systems, which do not match the conduct of experienced drivers. An autonomous vehicle driving alongside pedestrians should convey a natural and a sociallyaware behaviour. Therefore, the vehicle should not merely react to the behaviour of the surrounding agents, but should rather cooperate and proactively interact with its surrounding. Excluding this aspect from the navigation scheme results in over-reactive behaviours, an unnatural driving pattern and a suboptimal navigation solution. This paper presents a proactive longitudinal velocity control method, appropriate for navigation in close interaction with pedestrians. The work uses a cooperation-based pedestrians-vehicle behavioural model to find the optimal longitudinal velocity control. The method is implemented in lateral crossing scenarios with a dense crowd of pedestrians. The results are then compared with a reactive navigation system. The method is evaluated in terms of the vehicle's travel time and the safety of the pedestrians in the scene.
\end{abstract}

\section{INTRODUCTION}

Despite the ongoing advancements in the field of autonomous transportation, a full autonomy (level 5) is still not achieved in any current driving system [1]. Studies show that the major challenges on the way to full autonomy lie in pedestrian populated environments, where new ethical, social and legal considerations arise [2], [3]. This served as a motivation for researches and stakeholders in the field to study pedestrians-vehicle interaction and develop sociallyaware navigation systems. Primary works on socially-aware navigation were focused on pedestrian sized robotic applications. New navigation polices were introduced by using an interaction model [4]-[6]. Autonomous vehicles navigation systems benefited from these solutions later on. However, more recent studies argue that a different adaptation is required for pedestrians-vehicle interaction [7]-[9]. Each of these studies developed an interaction model adapted to their suggested interaction scenarios. Two of these models are based on social and psychological traits [7], [8] and one extends the social force model (SFM) by adding new forces specific to the vehicle's influence [9]. These models can be used to develop socially-aware navigation systems suitable for autonomous vehicles. However, the main limitation in the state of the art in socially-aware navigation is the lack of proactivity. Proactive behaviours are natural behaviours

\footnotetext{
${ }^{1}$ Univ. Grenoble Alpes, Inria, 38000 Grenoble, France

${ }^{2}$ Univ. Cote d'Azur, Inria, Sophia Antipolis, France
}

applied by drivers in everyday scenarios. Expert drivers interact, cooperate and influence other road users to navigate in an optimal manner. Applying this kind of proactivity in autonomous driving systems is key to a natural and socially acceptable behaviour. Moreover, the advantages of a proactive navigation is particularly prominent in dense pedestriansvehicle interaction scenarios. A reactive controller cannot consider the cooperation of the pedestrians in the scene and their reactions to the vehicle. This leads to over penalizing the vehicle's navigation options. Subsequently, the reactive controller would have a poor performance in such scenarios [10], leading to suboptimal navigation solutions or even the freezing of the vehicle in some cases. Although the term "Proactivity" is not used explicitly in the literature very often, the concept is considered in several applications. These applications include tasks which require influencing the work space. To cite a few Example, this can be a leader/follower task such as the work in [4], or minimizing the social effect of the navigation policy in [11]. In this work, the proactivity is considered as an invitation to the pedestrians to cooperate with the vehicle and change their planned paths.

To investigate the pros and cons of a proactive navigation system, this study targets a specific pedestrians-vehicle interaction scenario in shared spaces. A shared space does not apply any restrictions or priority rules on the vehicle's navigation policy. The navigation system of the vehicle consists of a global $A^{*}$ planner and a local proactive longitudinal velocity controller. The study analysis the performance of the controller in a lateral crossing scenario. The proactive solution suggested in this work is compared to a reactive navigation method. The performance is evaluated based on the vehicle's travel time and the safety of the pedestrians in the scene. It is important to point out that comparing solely based on the longitudinal control limits the performance of both the proactive and reactive controllers. However, the choice of the lateral crossing scenario with a dense crowd is made to compensate for this limitation, as the changes in the longitudinal velocity is more important that the lateral changes in such scenarios.

The structure of this paper is as follows: Section II explains the models of both the pedestrian, the vehicle and the interaction model between the two. Section III deals with the calculations of the collision probability, while section IV presents the proactive navigation framework. Finally, section $\mathrm{V}$ discusses the main results of this work. 


\section{Pedestrians And Vehicle Modeling}

A proactive navigation scheme is specific to pedestriansvehicle interaction scenarios. Therefore, it is essential to clearly understand the model of a pedestrian, that of the vehicle and eventually the model of interaction between the two. The models explained in this section are used when referring to a pedestrian or a vehicle throughout this study.

Navigating among pedestrians imposes low velocities and acceleration limits on the vehicle. In this case, the vehicle is modelled using the kinematic bicycle model with a zero slip assumption [12]. The position of the center of mass of the vehicle and its orientation at time $t$ is $X_{V}(t)=$ $\left[x_{v}(t), y_{v}(t)\right]^{T}$ and $\theta_{v}(t)$ respectively. The steering angle is $\delta(t)$ and the longitudinal velocity control is $u(t) . L$ is the length of the vehicle. Finally, the differential model of the vehicle is:

$$
\begin{aligned}
& \dot{X_{V}}(t)= {\left[u(t) \cos \theta_{v}(t), u(t) \sin \theta_{v}(t)\right]^{T} } \\
& \dot{\theta}_{v}(t)=\frac{u(t)}{L} \tan \delta(t)
\end{aligned}
$$

On the other hand, a pedestrian is modelled as a point in the $2 D$ plane. The position of a pedestrian $j$ at time $t$ is $X_{j}(t)=\left[x_{j}(t), y_{j}(t)\right]^{T}$ and its velocity is $V_{j}(t)=$ $\left[v_{x_{j}}(t), v_{y_{j}}(t)\right]^{T}$. Two types of interactions should be considered to model a pedestrian: the interactions with other pedestrians in the scene and the interactions with the vehicle. The cooperation-based trajectory planning model presented in [7] suggested a suitable interaction model. In a first step, the model assigns a cooperation factor $\left(C F_{j}(t)\right)$ to each pedestrian $j$ at time $t$. This factor is a quantitative measure of the pedestrian's tendency to cooperate with the vehicle. This measure is based on the state of the space, the goal of the pedestrian and the behaviour of the vehicle. The latter is introduced using the measure of the probability of collision between the pedestrian and the vehicle. Once this factor is determined, another cooperation-based trajectory planning model is ran to plan the pedestrian's path. The global framework of the model is illustrated in Fig. 1

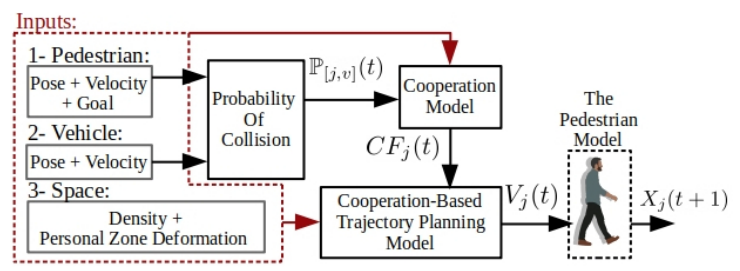

Fig. 1. The cooperation-based pedestrian's trajectory planning model

However, one drawback of this model, pointed out in [7], is that the model is impersonal. Meaning that two pedestrians existing in the same situation with the same goal destination will act similarly. Which is an incorrect assumption in real life situations. Multiple other factors can affect the behaviour of a pedestrian. These factors can include the age, physical and psychological state of the pedestrian for example [13]. Excluding these factors would lead to errors or biases in the trajectory prediction. To account for these factors, a new parameter is added to the cooperation model. We call it the inner cooperation factor $(I C F)$. This parameter is unique to each agent and constant throughout an interaction. The $I C F$ replaces the additive parameter $B$ in the cooperation model as follows: The cooperation model of an agent $j$ as shown in [7] is: $C F_{j}(t)=A I+B$, where $I \in[0,1]^{4 \times 1}$ is the model inputs and $A \in \mathbb{R}^{1 \times 4}, B=0.5$ are the model parameters. Then, the modified cooperation model is:

$$
C F_{j}(t)=A I+I C F \quad \text { with: } I C F \in[0,1]
$$

During an interaction scenario, the $I C F$ of each detected pedestrian is updated over consecutive time intervals (Fig. 2). First, an initial value is assumed $(I C F=B)$. Then, the trajectory of the agent is predicted for a fixed prediction horizon $T_{h}$ (using the previous model, as shown in Fig. 11). This is then compared with the actual observed trajectory. $I C F$ is updated using a simple gradient descent method to minimize the trajectory prediction error. The computation of the gradient of the error is straightforward since both the modified cooperation model and the trajectory planning model are linear.

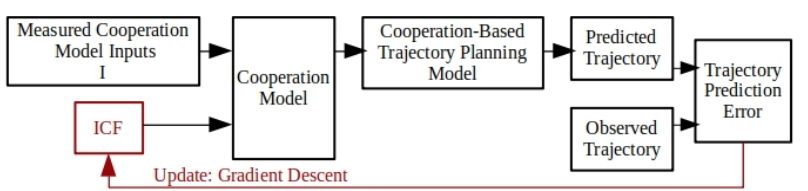

Fig. 2. Updating the inner cooperation factor

\section{The Probability Of Collision}

The probability of collision (POC) between the vehicle and a pedestrian is the main parameters expressing the effect of the vehicle's behaviour on the cooperation of the pedestrian in the interaction model [7]. Therefore, the proactive control can be derived using the gradient of the POC to convey a desired vehicle's influence. Most of the methods used to compute the POC between two entities are numerical and evaluating the gradient is not straightforward [14]. This is a drawback in our case. Therefore, our selected method depends on using an analytical form with a differentiable function. The method is based on using a probability distribution to describe the trajectory of each agent. This is used to derive a probability density function (PDF) of the Euclidean distance between the two agents. Finally, the POC is equal to the probability of the distance falling below a threshold minimum distance.

Given a pedestrian $j$ in interaction with a vehicle. Assuming that each of the position parameters for both the pedestrian and the vehicle follow a natural distribution with a variance $\sigma^{2}(t)$, then:

$$
E_{x}(t)=\left(x_{j}(t)-x_{v}(t)\right) \sim \mathcal{N}\left(\mu_{x_{j}}(t)-\mu_{x_{v}}(t), 2 \sigma(t)\right)
$$

where the symbol $\mu_{[X]}$ denotes the mean value of the parameter $[X]$.

Then, the weighted distance between the pedestrian and the 
vehicle follows a two degrees of freedom non-central $\mathcal{X}$ distribution [15]:

$$
\begin{gathered}
Z(t)=\frac{\sqrt{E_{x}^{2}(t)+E_{y}^{2}(t)}}{2 \sigma_{x}(t)} \sim \mathcal{X}(2, \lambda(t)) \\
\text { with: } \lambda(t)=\frac{D(t)}{2 \sigma_{x}(t)}
\end{gathered}
$$

where $D(t)$ is the Euclidean distance between the mean values of the positions of the pedestrian and the vehicle at time $t$.

The probability of collision at a time instant $t_{0}$ is computed for a fixed prediction horizon $T_{h}$. First, the trajectories of both agents are predicted over the fixed time interval $\left[t_{0}, T_{h}+t_{0}\right]$. Then, the distribution of the weighted distance between the two agents $(Z)$ is estimated over this time interval. Finally, the probability of collision is computed using the probability density function of the weighted distance $\left(f_{Z}(x ; 2, \lambda)\right)$ and a limit minimum distance $d_{\text {lim }}$ as follows:

$$
\mathbb{P}_{[j, v]}\left(t_{0}\right)=\frac{1}{T_{h}} \int_{t=t_{0}}^{t_{0}+T_{h}} \int_{x=0}^{d_{l i m}} f_{Z}(x ; 2, \lambda(t)) d x d t
$$

\section{Proactive Longitudinal Velocity Control}

Ensuring the safety of the pedestrians in the scene remains the main priority of the navigation system. Invoking more cooperative pedestrian behaviours for is desired providing that it does not compromise the safety at any time. The suggested safety index (SI) is a quantitative measure based on a minimal distance between the vehicle's body and the pedestrian. Using the concepts of the personal and the cooperation zones discussed in [7], the safety index for a pedestrian $j$ at time $t$ is evaluated as follows:

$$
S I_{j}(t)=\frac{D_{j}(t)-R_{S}}{R_{C}-R_{S}}
$$

where: $D_{J}$ is the minimum distance between the pedestrian $j$ and the body of the vehicle, $R_{S}$ is the radius of the personal zone and $R_{C}$ is the radius of the cooperation zone (Fig. 3 B). SI takes negative values when the vehicle enters the personal zone of the pedestrian indicating a failed navigation. Entering the cooperation zone results in $S I_{j}<1$ expressing a possible discomfort of the pedestrian. A better navigation policy results in larger SI values.

Moreover, to reduce the complexity of the navigation task, a zone of vehicle's influence is defined. In the navigation policy, only pedestrians detected within this zone are taken into consideration. The influence zone proposed in this work is a $180^{\circ}$ zone with the same orientation as the vehicle. The radius of the zone is proportional to the linear velocity of the vehicle. This ensures a larger influence margin in higher velocities. The parameters of the influence zone (center $X_{I}=\left[x_{I}, y_{I}\right]^{T}$ and radius $R_{I}$ ) are found as follows:

$$
X_{I}(t)=X_{V}(t)-\frac{L}{2} V(t), \quad R_{I}(t)=r_{\min }(1+u(t))
$$

with: $r_{\min } \geq 0, V(t)=\left[u(t) \cos \theta_{V}(t), u(t) \sin \theta_{V}(t)\right]^{T}$.

Fig. 3 A shows multiple examples of the influence zone with different velocities of the vehicle.

In the following, the problem of proactive navigation is presented in a pedestrians-vehicle interaction scenario. The problem is formulated as an optimal control problem to find the longitudinal velocity control of the vehicle. The solution is then found based on the pedestrian-vehicle cooperation model.

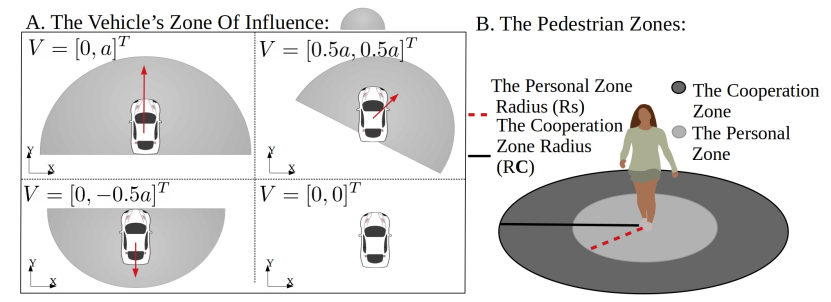

Fig. 3. A: The zone of influence of the vehicle with different vehicle velocities, where $a \in \mathbf{R}_{+}, \mathrm{B}$ : The pedestrian zones used to evaluate the security index

\section{A. Problem Formulation}

Given an interaction scenario between a vehicle and $N$ pedestrians. Let $X_{P}(t)=\left[X_{1}(t), \ldots, X_{N}(t)\right], V_{P}(t)=$ $\left[V_{1}(t), \ldots, V_{N}(t)\right]$ be the perceived positions and velocities of the $N$ pedestrians at time $t$ respectively. Given that only the first $M \leq N$ pedestrians are detected in the influence zone of the vehicle. Provided with a steering angle from a higher level global planner, find the longitudinal velocity control $u(t)$ of the vehicle, such that:

$$
\begin{aligned}
& u(t)=\min _{u(t) \in\left[-u_{\max },+u_{\max }\right]} J_{\text {reg }}\left(u(t), V_{P}(t), X_{P}(t), X_{V}(t)\right) \\
& J_{\text {reg }}=\frac{1}{T_{h}} \int_{t=t_{0}}^{t_{0}+T_{h}} J\left(u(t), V_{P}(t), X_{P}(t), X_{V}(t)\right)+T(u(t))
\end{aligned}
$$

where $u_{\max }$ is the speed limit of the vehicle and $J$ is a cost function that aims to maximize the cooperation of the influenced agents while ensuring their safety:

$$
J=\frac{1}{M} \sum_{j=1}^{M} \alpha_{1}\left(1-C F_{j}(t)\right)-\alpha_{2} S I_{j}(t)
$$

with $\left(\alpha_{1}, \alpha_{2}\right) \in \mathbb{R}_{+}^{2}$, and $T(u(t))$ is a Tikhonov regularization function [16] added to penalize infinite solutions and prefer solutions with smaller $L_{2}$ norms:

$$
T(u(t))=\alpha_{3}\|u(t)\|_{2}^{2} ; \quad \alpha_{3} \in \mathbb{R}_{+}
$$

\section{B. Solving the Optimal Control Problem}

The solution for the optimization problem in Eq. 8 derived using the Hamilton-Jacobi-Bellman (HJB) equation has the form:

$$
u(t)=\frac{1}{2 M \alpha_{3}} \sum_{j=1}^{M} \alpha_{1} \partial_{u} C F_{J}(t)+\alpha_{2} \partial_{u} S I_{j}(t)
$$

the symbol $\partial_{y} x$ is equivalent to $\frac{\partial x}{\partial y} ; \forall x, y$.

The problem is then reduced to finding the gradient of the cooperation factor and the safety index with respect to the vehicle's control.

The gradient of the safety index of agent $j$ depends only on the gradient of the distance to the vehicle $D_{j}(t)$ (Eq. 6 . 
To derive the gradient of the distance, let's rewrite both the pedestrian's and the vehicle's differential models. Using the same parameter assumptions used in section II for both the pedestrian and the vehicle, let's write: $X_{u}^{V}=\partial_{u} X_{V}$ and $X_{u}^{j}=\partial_{u} X_{j}$. Then, the gradient of the Euclidean distance can be written as:

$$
\partial_{u} D_{j}(t)=\partial_{X_{P}} D_{j}(t) X_{u}^{j}+\partial_{X_{V}} D_{j}(t) X_{u}^{V}
$$

The term $X_{u}^{V}$ is obtained by a numerical solution of the vehicle's model. The only term left unknown is $\dot{X}_{u}^{j}=$ $\partial_{u} V_{j}(t)$ which can be determined using the cooperationbased pedestrian trajectory planning model [7], and the following two gradients can be derived by simple calculations:

$$
\begin{array}{r}
\partial_{u} V_{j}(t)=\mathbf{F}_{1}\left(\partial_{u} V_{j}(t-1), C F_{j}(t), \partial_{u} \mathbb{P}_{[j, v]}(t)\right) \\
\partial_{u} C F_{j}(t)=\mathbf{F}_{2}\left(\partial_{u} V_{j}(t-1), \partial_{u} \mathbb{P}_{[j, v]}(t)\right)
\end{array}
$$

where: $\mathbf{F}_{1}: \mathbb{R}^{3} \rightarrow \mathbb{R}^{2}, \mathbf{F}_{2}: \mathbb{R}^{2} \rightarrow \mathbb{R}$ are linear functions.

Deriving the probability of collision using the method in section III allows to compute its gradient using the probability density function of the distance $f_{Z}\left(d_{l i m} ; 2, \lambda\right)$ :

$$
\begin{gathered}
\partial_{u} \mathbb{P}_{[j, v]}(t)=\frac{1}{d_{l i m}} \partial_{u} D_{j}(t) f_{Z}\left(d_{l i m} ; 2, \lambda\right) \\
\text { V. Simulations AND Results }
\end{gathered}
$$

\section{Simulations and Results}

The test simulations include interactions between the vehicle and a group of pedestrians in a lateral crossing scenario. The pedestrians in the simulations plan their trajectories using the cooperation-based trajectory planning model with the modified cooperation factor, as illustrated in Fig. 1. In every simulation, the pedestrians are initialized with random inner cooperation factors $(I C F \in[0,1])$ and random goal points within a defined workspace.

Fig. 4 shows the overall structure of the vehicle's proactive navigation system.

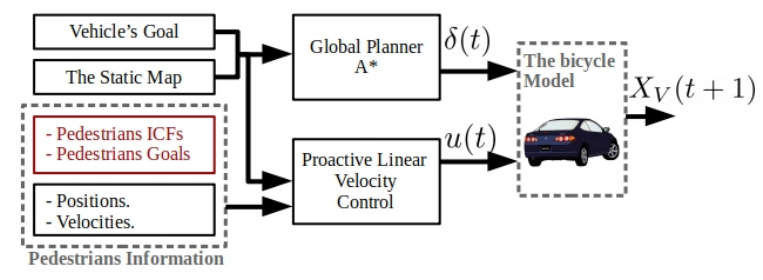

Fig. 4. The Proactive Navigation Policy

\section{A. A reactive method and the possible freezing problem}

The same previous structure is used to run the simulations with a reactive linear velocity controller. The reactive control is computed using the same method as the proactive one, with a modified cost function. This modified cost function includes only a reactive term based on the safety index:

$$
J_{\text {reactive }}(t)=\frac{1}{M} \sum_{j=1}^{M}-\alpha_{2}^{\prime} S I_{j}(t)
$$

where $\alpha_{2}^{\prime} \in \mathbb{R}_{+}, M$ is the total number of pedestrians detected in the influence zone and $S I_{j}$ is the same safety index defined in Eq. 6

To compare the two methods, the same simulation is ran twice. With the same initial positions and goals for both the vehicle and the pedestrians. As well as, the same inner cooperation factors $(I C F \mathrm{~s})$ for the pedestrians. The only difference is the local velocity controller (proactive or reactive).

An example simulation is shown in Fig. 5. The simulation includes a lateral crossing scenario between a vehicle and a flow of $N=66$ pedestrians. The maximum velocity allowed in the space is $4 m . s^{-1}$ for the vehicle and $3 m . s^{-1}$ for the pedestrians. The $I C F \mathrm{~s}$ for the pedestrians are assigned randomly between $[0,1]$. The figure shows the resulting trajectories in the $X Y$ plane with the two control modes. In each mode, four different screenshots of the simulation are shown for a better visualization of its progress. The time interval present in each screenshot is noted on the right of the figure. The figure clearly shows that in the proactive mode the vehicle successfully crosses the pedestrians flow by anticipating their cooperation. While in the reactive mode, the vehicle stops to ensure the safety margins. Once the vehicle stops, the pedestrians continue their crossing and the reactive mode will not be able to find a valid solution. This results in the freezing of the vehicle until the flow of pedestrians ends.

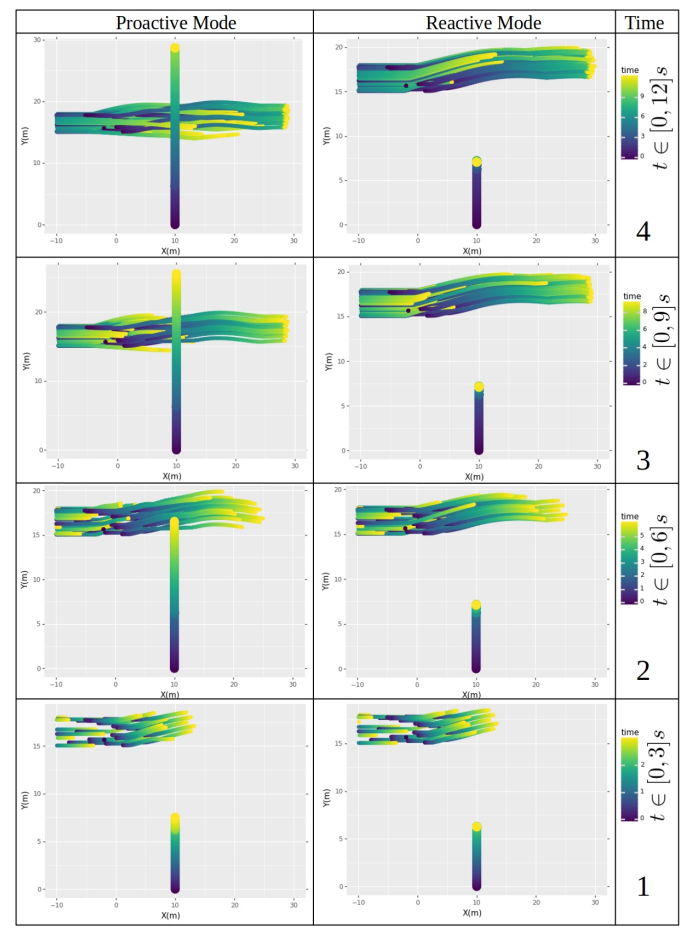

Fig. 5. Pedestrian-vehicle lateral crossing simulation (two modes)

\section{B. The Vehicle's Travel Time}

The travel time of an agent (vehicle or pedestrian) is the time required to travel from the initial position to the goal 
destination. To compare the travel time between the proactive mode and the reactive mode in relative terms, we define two parameters. These two parameters serve as reference travel times. The first parameter is the reference pedestrian crossing time $\left(T T_{\text {peds }}\right)$ : the time required for all the pedestrians in the simulation to complete their crossing without a vehicle interference. This time is measured by running a reference simulation which contains the same pedestrian configuration, but without the vehicle. The second parameter is the vehicle's reference travel time $\left(T T_{\text {ref }}\right)$ : the time required for the vehicle to reach its final destination without any pedestrian interference. In the same manner, this time is measured by running a simulation which does not contain any pedestrians but has the same vehicle initialization and goal.

Let $T T(s)$ be the travel time of the vehicle in a simulation $s$ regardless of the navigation mode. Then, we can define two relative travel times for the vehicle in this simulation $s$ :

- The vehicle's travel time relative to its reference travel time:

$$
T T_{[v e h / r e f]}(s)=\frac{T T_{r e f}(s)-T T(s)}{T T_{r e f}} \times 100 \%
$$

- The vehicle's travel time relative to the pedestrians' refernce crossing time:

$$
T T_{[v e h / p e d s]}(s)=\frac{T T_{p e d s}(s)-T T(s)}{T T_{p e d s}} \times 100 \%
$$

Using these two relative terms instead of the original vehicle travel time in seconds (TT $(s)$ ), allows us to compare the performance of the proactive and reactive modes independently of the pedestrians' configuration in a set of test simulations.

A total of 200 simulations are run (100 pair) with a random initialization. Each pair consists of the same simulation with a different navigation mode (reactive / proactive). The two previous relative travel times are computed for every simulation. Fig. 6A shows the histogram of the vehicle's travel time relative to its reference travel time, where the two modes are shown in different colors. $T T_{[v e h / r e f]}$ takes negative values in both modes. Which shows that even the proactive mode slows down the vehicle or even immobilise it sometimes to cross the pedestrian flow. However, this delay is much smaller in the proactive case. The proactive mode yields a navigation $10-50 \%$ slower than the reference crossing. While the reactive mode can results in very high delays (can be over $250 \%$ slower). The histogram of the vehicle's travel time relative to the pedestrians reference crossing time is shown in Fig. 6.B. $T T_{[\text {veh } / \text { peds] }}$ takes positive values for the proactive mode and negative values for the reactive mode. This means that the proactive mode finds a solution while the pedestrians are still crossing. Therefore reaching the goal destination before all the pedestrians have crossed. While in the reactive mode the vehicle is frozen for some time due to the detection of nearby pedestrians. The important point to notice here is the range of values existent in the histogram of $T T_{[v e h / p e d s]}$. In the reactive mode, the histogram is extended over a very small range (all values are around $-20 \%$ ). This shows that there is a high correlation between the pedestrian crossing time and the vehicle's travel time in the reactive mode. On the other hand, $T T_{[v e h / \text { peds }}$ in the proactive mode takes a large range of values $([15 \%, 60 \%])$. This shows that the proactive mode does not depend on the crossing time of the pedestrian flow. It also shows that the travel time gain in the proactive mode differs depending on the particular interaction situation. Because the proactive action is only taken when the safety of the pedestrians is ensured. In other words, in the proactive mode a solution is always found regardless of the crossing time of the pedestrian flow. However, a large gain in the travel time cannot always be ensured. A more detailed analysis of our simulation results show that the gain in the travel time is larger with larger crowds of pedestrians.

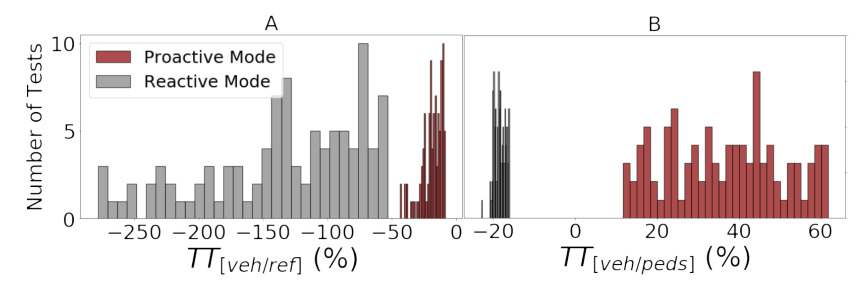

Fig. 6. Histogram of the vehicle's relative travel time.

\section{Pedestrians' safety test}

To evaluate the navigation method from a safety perspective, a global performance index (PI) is defined in a simulation of period $T$ with $N$ pedestrians as:

$$
P I=\min _{j \in[1, \ldots, N]} \min _{t \in[0, T]} S I_{j}(t)
$$

where $S I_{j}(t)$ is the safety index defined in Eq. 6 for a pedestrian $j$ at time $t$.

In other words, the performance of the vehicle is judged by the minimum safety margin it has with a pedestrian over the simulation time (i.e. worst case margin).

Fig. 7 shows the histogram of the performance index. $P I$ is evaluated over the same previous 100 simulations with the proactive mode. The histogram shows that most $P I$ values are in $[0,1]$. This means that in the proactive mode the vehicle enters the cooperation zone of at least one pedestrian at a certain time. However, the histogram does not contain any negative values. This mean that the proactive mode maintained the imposed safety margins and the vehicle did not enter the security zone of any pedestrian. Therefore, the navigation was successful in terms of safety.

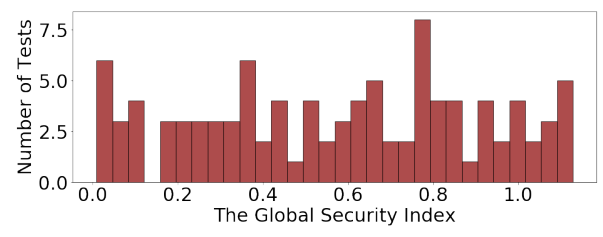

Fig. 7. The histogram of the global security index $P I$

However, the current safety measure uses only the position of the pedestrian with the assumption of equal safety margins 
around the pedestrian. This is a strict measure in comparison with real behaviours of pedestrians. A safety measure that considers the velocity of the pedestrian along with a different security zone (ellipses instead of a circle for example [17]) is required for a more realistic estimation.

\section{Results with more/less cooperative crowds}

To investigate the effect of the cooperativeness of the crowd, the $I C F$ parameter is manipulated. By initializing the pedestrians' $I C F$ s values in $[0.5,1]$, a more cooperative crowd is simulated. Whereas, a less cooperative crowd is simulated by initializing it in $[0,0.5]$. In the previous test, the $I C F$ s for the pedestrians are chosen randomly in $[0,1]$ to simulate a normal mixed crowd.

200 more simulations are ran in the proactive mode of pedestrians-vehicle lateral crossing scenario. Combined with the previous tests, this results in a total of 300 proactive mode simulations: 100 with a normal crowd, 100 with a cooperative crowd and 100 with an uncooperative crowd. The vehicle's travel time relative to its reference travel time $T T_{[v e h / r e f]}$ is computed for every simulation. Fig. 8 shows the normal distribution fit to the histogram of $T T_{[v e h / r e f]}$ for each type of the simulated crowd. The figure shows that the proactive mode does depend on the cooperativeness of the simulated crowd. A better performance is obtained with more cooperative crowds. Whereas, longer time delays can occur with highly uncooperative crowds.

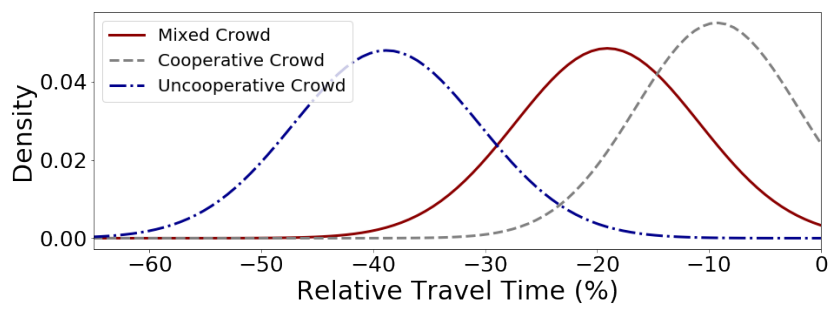

Fig. 8. Normal distribution of the vehicle's relative travel time $\left.T T_{[v e h / r e f}\right]$

\section{CONCLUSION}

This paper presents a navigation solution suitable to pedestrians-vehicle interaction scenarios. This proactive solution helps the autonomous driving system to convey a more natural behaviour and avoid suboptimal navigation solutions. The work uses a cooperation-based behavioural model for the pedestrians-vehicle interaction [7]. The proactive framework is then built based on the cooperation measure. The longitudinal velocity is controlled locally to increase the cooperativeness of the pedestrians in interaction with the vehicle. This is done while ensuring (and prioritizing) the safety of all the pedestrians in the scene. The results of the proactive navigation is compared to a reactive navigation mode. The results shows a significant travel time gain with the proactive mode regardless of the density of the pedestrians crowd. This gain is obtained while ensuring the safety measures. The performance of the method was analysed with different types of pedestrian crowds in term of their cooperativeness. This work serves as a starting point to build a complete proactive navigation framework. The scope of this study only covered the longitudinal velocity control in lateral crossing scenarios. This is a limitation that we will address in future works to target both the longitudinal and lateral controls. In addition, more complex scenario will be considered to simulate more realistic everyday scenarios.

\section{ACKNOWLEDGMENT}

This research is part of the HIANIC project, funded by the French National Research Agency (ANR-17-CE22-0010).

\section{REFERENCES}

[1] E. Ackerman, "Toyota's gill pratt on self-driving cars and the reality of full autonomy," Spectrum, International Institute of Electrical Engineers (www.ieee.org), Jun. 2017.

[2] T. Bellet et al., "From semi to fully autonomous vehicles: New emerging risks and ethico-legal challenges for human-machine interactions," Transportation Research Part F: Traffic Psychology and Behaviour, Elsevier, vol. 63, pp. 153-164, Apr. 2019.

[3] L. Adouane, "Toward fully autonomous vehicle navigation: From behavioral to hybrid multi-controller architectures," in The 11th International Workshop on Robot Motion and Control (RoMoCo), Wasowo, Poland, Jul. 2017, pp. 85-98.

[4] A. Clodic et al., "Rackham: An interactive robot-guide," in The 15th IEEE International Symposium on Robot and Human Interactive Communication, Sep. 2006, pp. 502-509.

[5] G. Ferrer, A. Garrell, and A. Sanfeliu, "Robot companion: A socialforce based approach with human awareness-navigation in crowded environments," in IEEE/RSJ International Conference on Intelligent Robots and Systems, Tokyo, Nov. 2013, pp. 1688-1694.

[6] Y.-Q. Jiang, B.-K. Chen, B.-H. Wang, and W.-F. Wong, "Extended social force model with a dynamic navigation field for bidirectional pedestrian flow," Frontiers of Physics, vol. 12, pp. 1-9, May 2017.

[7] M. Kabtoul, A. Spalanzani, and P. Martinet, "Towards proactive navigation: A pedestrian-vehicle cooperation based behavioral model," International Conference on Robotics and Automation, Paris, France, May 2020.

[8] T. Randhavane, A. Bera, E. Kubin, A. Wang, K. Gray, and D. Manocha, "Pedestrian dominance modeling for socially-aware robot navigation," CoRR, vol. abs/1810.06613, 2018.

[9] D. Yang, U. Ozguner, and K. Redmill, "Social force based microscopic modeling of vehicle-crowd interaction," in IEEE Intelligent Vehicles Symposium, Jun. 2018, pp. 1537-1542.

[10] A. Vemula, K. Muelling, and J. Oh, "Modeling cooperative navigation in dense human crowds," in 2017 IEEE International Conference on Robotics and Automation, Singapore, May 2017, pp. 1685-1692.

[11] G. Ferrer and A. Sanfeliu, "Proactive kinodynamic planning using the extended social force model and human motion prediction in urban environments," in IEEE/RSJ International Conference on Intelligent Robots and Systems, Sep. 2014, pp. 1730-1735.

[12] P. Polack, F. Altché, B. Novel, and A. de La Fortelle, "The kinematic bicycle model: A consistent model for planning feasible trajectories for autonomous vehicles?" in IEEE Intelligent Vehicles Symposium, Jun. 2017, pp. 812-818.

[13] F. Durupınar, U. Güdükbay, A. Aman, and N. I. Badler, "Psychological parameters for crowd simulation: From audiences to mobs," IEEE Transactions on Visualization and Computer Graphics, vol. 22, no. 9, pp. 2145-2159, Sep. 2016.

[14] L. Chen, X.-Z. Bai, Y.-G. Liang, and K. Li, Calculation of Collision Probability. Springer, 12 2017, pp. 135-183.

[15] F. K. Chan, "Miss distance-generalized variance non-central chi distribution," in AAS/AIAA Space Flight Mechanics Meeting, 2011, pp. 11-175.

[16] A. N. Tikhonov, "On the stability of inverse problems," Doklady Akademii Nauk SSSR, vol. 39 (5), p. 195-198, 1943.

[17] J. Rios-Martinez, A. Spalanzani, and C. Laugier, "From proxemics theory to socially-aware navigation: A survey," International Journal of Social Robotics, vol. 7, pp. 137-153, Apr. 2014. 